\title{
Fibrinogen-Induced Regeneration Sealing Technique (F.I.R.S.T.). An Improvement and Modification of Traditional GBR: A Report of Two Cases
}

ISSN: 2637-7764

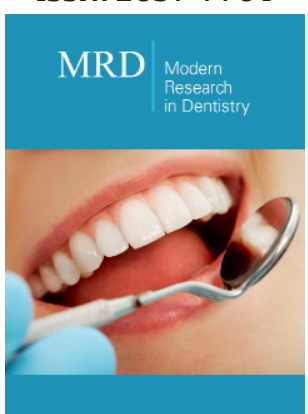

*Corresponding author: Foti Vincenzo, Private practice, Piazza Borgo Pila 40, Genova, Italy

Submission: 㘹June 09, 2020

Published: 㭗July 08, 2020

Volume 5 - Issue 2

How to cite this article: Foti Vincenzo, Rossi Roberto. Fibrinogen-Induced Regeneration Sealing Technique (F.I.R.S.T.). An Improvement and Modification of Traditional GBR: A Report of Two Cases. Mod Res Dent. 5(2). MRD.000609. 2020 DOI: 10.31031/MRD.2020.05.000609

Copyright@ Foti Vincenzo, This article is distributed under the terms of the Creative Commons Attribution 4.0 International License, which permits unrestricted use and redistribution provided that the original author and source are credited.

\author{
Foti Vincenzo* and Rossi Roberto \\ Private practice, Genova, Italy
}

\begin{abstract}
s
Guided bone regeneration is a technique widely known, clinicians know very well indications and limitations of this technique. One of the principles to achieve bone augmentation and formation resides in the stability of the blood clot forming under a barrier membrane. The technique proposed in this article has the goal of providing stabilization to the bone graft by adding fibrin sealant (FS) to the bone graft and also using the fibrin sealant to attach a bone membrane (cortical lamina) to the recipient site. This simple modification of the technique of guided bone regeneration is presented in two successful cases.
\end{abstract}

Keywords: Cortical lamina; Fibrinogen; Fibrin sealant; Guided bone regeneration; Implants

\section{Introduction}

\section{Fibrin Sealant}

Fibrin sealant (FS) is a medical device derived from human blood, used in surgery and in specialties since 1975 for his hemostatic and ahesive properties. The first application in the dental field dates to the 80', in oral surgery for those patients with disorders of hemostasis [1]. In periodontology Pini Prato GP et al [1]. in 1988 treated infra-bony defects by using FS to stabilize membranes in guided tissue regeneration procedures [2]. The first application in guided bone regeneration was reported by Wittkampf in 1989. He described a simple way to handle granules of HA in the reconstruction of narrow alveolar ridges using the FS to keep the particles together [3]. Hotz [4] used FS as a biological resorbable glue (thrombin was diluted to $1 \mathrm{U} / \mathrm{ml}$ ) in socket preservation.

The glue would keep the granules of HA in solid form until keratinized gingiva had sealed the socket [4]. Three years of clinical experience demonstrated the efficacy of the complex HA/FS in pre-prosthetic and reconstructive surgery [5]. The clinical study that demonstrated and a new peri-implant regenerative technique by adding the FS was the one of Corrente et al. [6]. The authors achieved supra-crestal regeneration without the use of a barrier membrane. The average vertical gain was $2.05 \mathrm{~mm}$ and histology showed newly formed trabecular bone [6]. Cardaropoli D et al. [7] 2013 modified the technique of Corrente by adding a collagen membrane, with this addition they reported a mean vertical gain of $3.95 \mathrm{~mm}$ [7]. In regenerative procedures, FS adds an excellent physical quality to the bone grafts, making them sticky, moldable and easy to adapt to local anathomy, without any waste of granules [8]. FS demonstrates an osteoinductive capacity when mixed with osteoconductive bio-materials [9-11]. Furthermore favors angiogenesis, thanks to the link of fibrinogen with VEGF growth factor, stimulating proliferation of endothelial cells [12]. Fibrinogen also induces formation of extracellular matrix to support blood supply, connective tissue and bone [13]. Linsley in 2012 demonstrated that fibrinogen induces growth of mesenchimal cells and their differentiation into osteoblasts [14]. One last effect of FS is to enhance and accelerate wound healing and closure [15]. 


\section{Cortical Lamina}

The cortical lamina (Cortical Lamina Osteobiol, by Tecnoss, Coazze, Italy) is a membrane used in GBR made of collagenated porcine bone. This membrane is very versatile because is produced in three different versions, curved, soft and bone layer. These three version satisfy all the different applications in GBR whether we are dealing with horizontal, vertical or three-dimensional defects [16]. Rossi et al. [17] carried out a clinical and histological study on humans, a series of patients with severely resorbed mandibles were first treated by means of the cortical lamina associated with collagenated porcine bone xenograft (GenOs Osteobiol By Tecnoss, Coazze, Italy) mixed with the patient's own blood clot. The lamina was positioned on top of the grafted edentulous ridge in the attempt of increasing the width and height. After healing periods of 6/8 months implants were inserted in the areas where the ridge augmentation took place, and careful biopsies were taken before inserting the fixtures.

All implants achieved osseointegration and up to one year after final restoration did not show any modification of the crestal level. Biopsies showed that all areas augmented had live mineralized bone [17]. If curved lamina because of its stiffness and flexibility can be adjusted to local anatomy just by means of sutures, the soft lamina behaves like any resorbable membrane but, being made of bone, maintains a certain elasticity and needs to be fixed by pins or tacks. Same can be said for the bone layer that needs screws to keep it in place. Cortical lamina proves to be a reliable device to perform ridge augmentation, the application of FS to the soft lamina became crucial in the development of the F.I.R.S.T. technique.

\section{F.I.R.S.T}

In 2016 authors found in the FS the ideal material to achieve a perfect fixation of the cortical lamina, preventing the use of screws. The objective was to create a system where the bone graft and the cortical lamina become one whole complex. Stability of the graft represents one of the key factors in GBR [18]. Aside from that the hypothesis was that FS would improve regeneration through the osteoinductive activity of fibrinogen. The name "FibrinogenInduced Regeneration Sealing Technique" object of this paper generated from this thought.

\section{Case Presentation I}

The patient was a woman 64-year-old with history of periodontal disease. Non-smoker, in good general health, she did not report allergies or intolerances to anesthetics and drugs. She presented with stage IV periodontitis and partial edentulism in the second quadrant with a horizontal bony deficiency. The first step represented full-mouth disinfection and systemic therapy with Metronidazole and Amoxicillin for 7 days to reduce the infection. After 3 months of good periodontal maintenance, the CBCT showed a defect where the thickness of the residual bone was less than $5 \mathrm{~mm}$. The thickness of the buccal plate prior to surgery was less than $1 \mathrm{~mm}$ while minimum thickness recommended from evidence should be $2 \mathrm{~mm}$ [19]. After local anesthesia with $4 \%$ Articaine with adrenaline 1:200,000, a crestal incision and a full-thickness vestibular flap with vertical incisions mesial to tooth 24 and distal at tuber level was elevated. A full-thickness palatal flap was also raised $10 \mathrm{~mm}$ apically without vertical incisions. After obtaining adequate passivation of the buccal flap, decortications of the buccal bone wall were performed using the IM1S PiezoSurgery insert (Mectron, Carasco, Italy). IK 3.8x13mm and IK 3.8x11mm implants (Resista Group, Verbania, Italy) were placed in the sites 25 and 26. There were't exposed threads, but the buccal bone wall was thin.

After hydration for 10 minutes in sterile saline solution, a $25 \times 25 \mathrm{~mm}$ cortical lamina of $0.5 \mathrm{~mm}$ thickness (Soft Fine Cortical Lamina Osteobiol By Tecnoss, Coazze, Italy) was cut to size and tested on the horizontal vestibular defect with extension to the palatal side. After 1:10 dilution of the thrombin component with sterile double distilled water, a few drops of FS (Tisseel, Baxter, Rome, Italy) were applied to a collagenated porcine graft (GenOs, Osteobiol by Tecnoss, Coazze, Italy). The dilution is always necessary to avoid the immediate polymerization of FS which would get fibrous encapsulation of the graft without integration in defect area [20]. The mixture was applied to the horizontal defect creating a new over contoured anatomy. After 5 minutes the graft, stable and well adapted to the defect (without waste of granules), achieves a consistency similar to play dough. The inner part of the cortical lamina was also covered with some FG than the membrane was applied to the area of augmentation. The lamina was firmly attached to the local anatomy without the need of pins or sutures.

The surgery ended with 5/0 Polypropylene horizontal mattress sutures (Aragò, Barcelona, Spain). Amoxicillin 500mg every 8 hours for 7 days, Ibuprofen $600 \mathrm{mgx} 3$ for 3 days, Chlorhexidine $0.20 \%$ rinses morning and evening for 7 days were prescribed as postoperative medication. Postsurgical CBCT demonstrates a buccal augmentation of $4.05 \mathrm{~mm}$. The sutures were removed after 2 weeks of uneventful post-operative course (Figure 1-7). Six months after surgery, the clinical aspect showed an excellent volume of convex shape and hard consistency. The CBCT highlighted a horizontal gain of $4.05 \mathrm{~mm}$ with initial mineralization. The day of re-entry the area displayed a new anatomy. The mesial implant was covered with hard tissue reproducing the shape of the cover-screw. A biopsy was taken and stored in 10\% buffered neutral formalin and sent for histological examination. Healing abutments were connected and soft tissue sutured in place. After 3 months a prosthetic restoration was performed by means of a gold-ceramic screwed bridge. The histological result showed newly formed trabecular bone, in close contact with residual particles and osteoid matrix where newly formed blood vessels and active osteoblasts were present. There were not inflammatory or necrotic cells. Three years after surgery, the clinical and CBCT control show hard and soft tissue stability. The horizontal measurement of new vestibular bone is stable at $4.05 \mathrm{~mm}$. The peri-apical rx shows no marginal bone loss. Four years after surgery, a significant finding was observed on the new CBCT: the graft appeared almost totally reabsorbed in favor of new bone which has a radiological quality similar to that of the native bone and there is a new mature cortical plate [21] similar to the palatal native bone (Figure 8-15). 


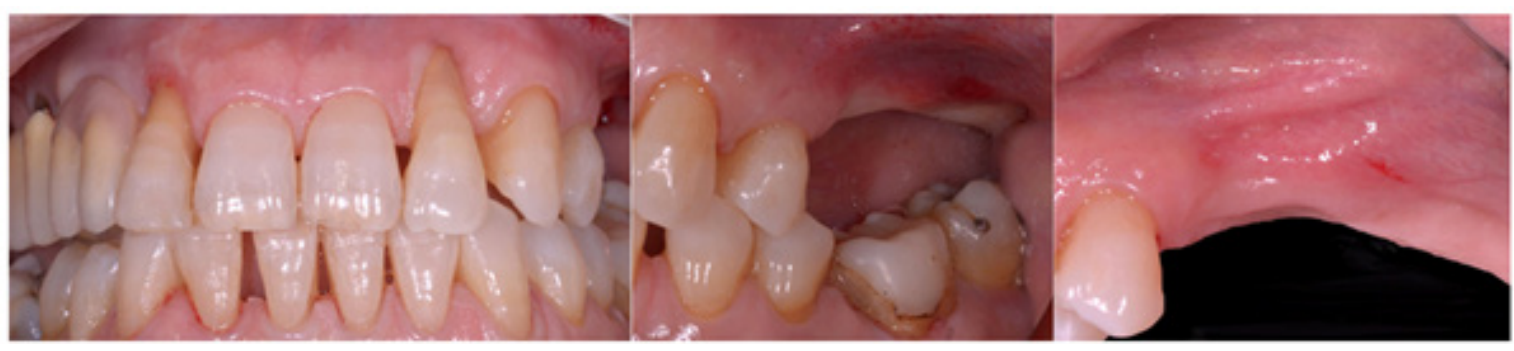

Figure 1: Initial status. Buccal plate concavity on the upper left quadrant.

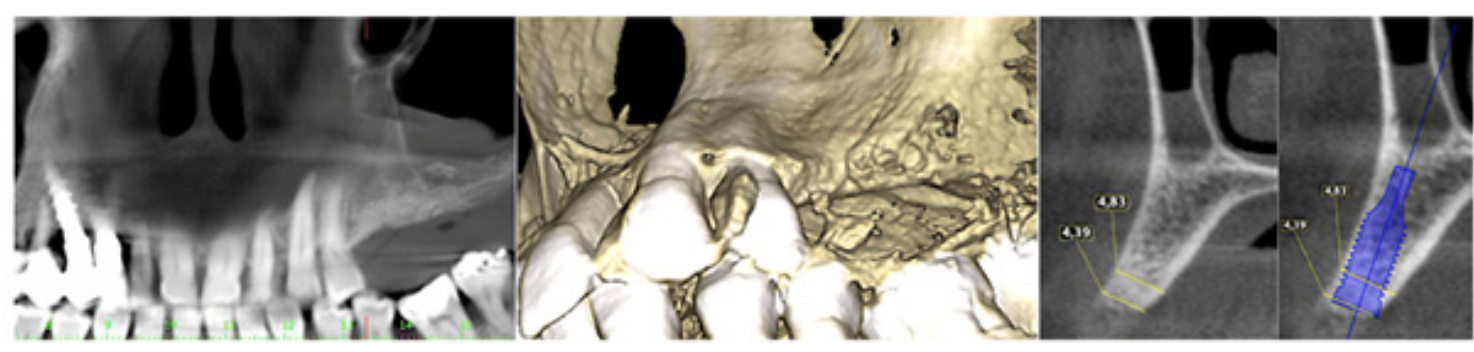

Figure 2: The thickness of the bone was less than $5 \mathrm{~mm}$. The buccal bone wall at planned implant position was less than $1 \mathrm{~mm}$ thick.

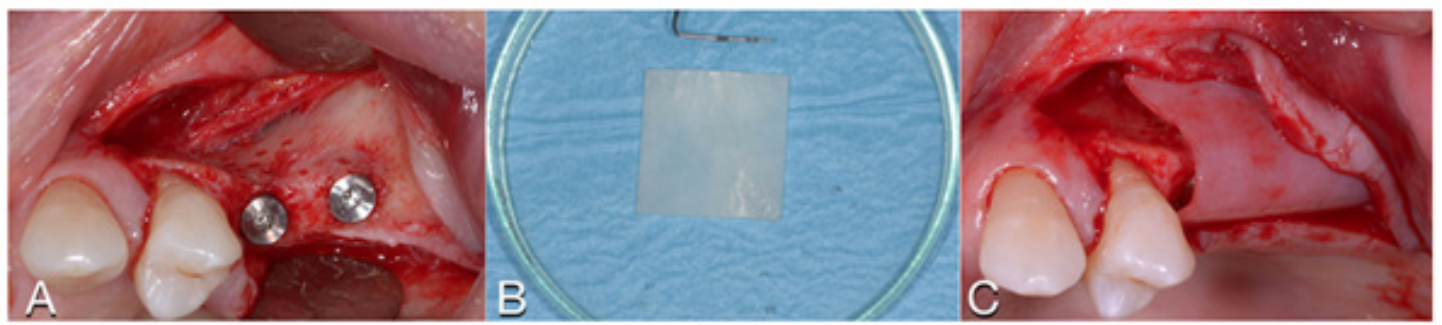

Figure 3: A. Buccal concavity. $\quad$ B. Soft Lamina Hydration. $\quad$ C. The membrane is elastic and shows tendency to open.

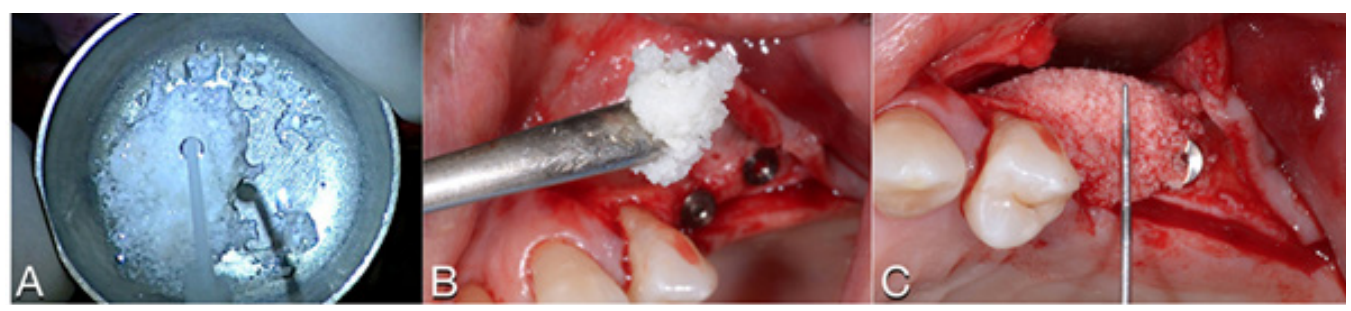

Figure 4: A. Tisseel mixed with graft. $\quad$ B. Graft adhesion. $\quad$ C. Graft volume and stability.

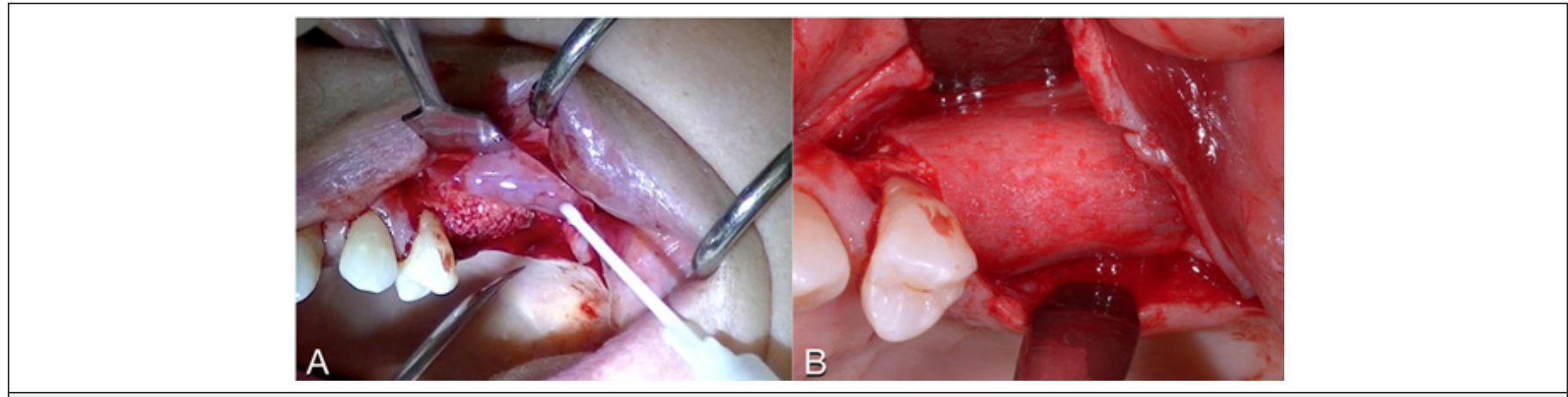

Figure 5: A. Tisseel applied to the lamina.

B. The lamina is stabilized without pins. 

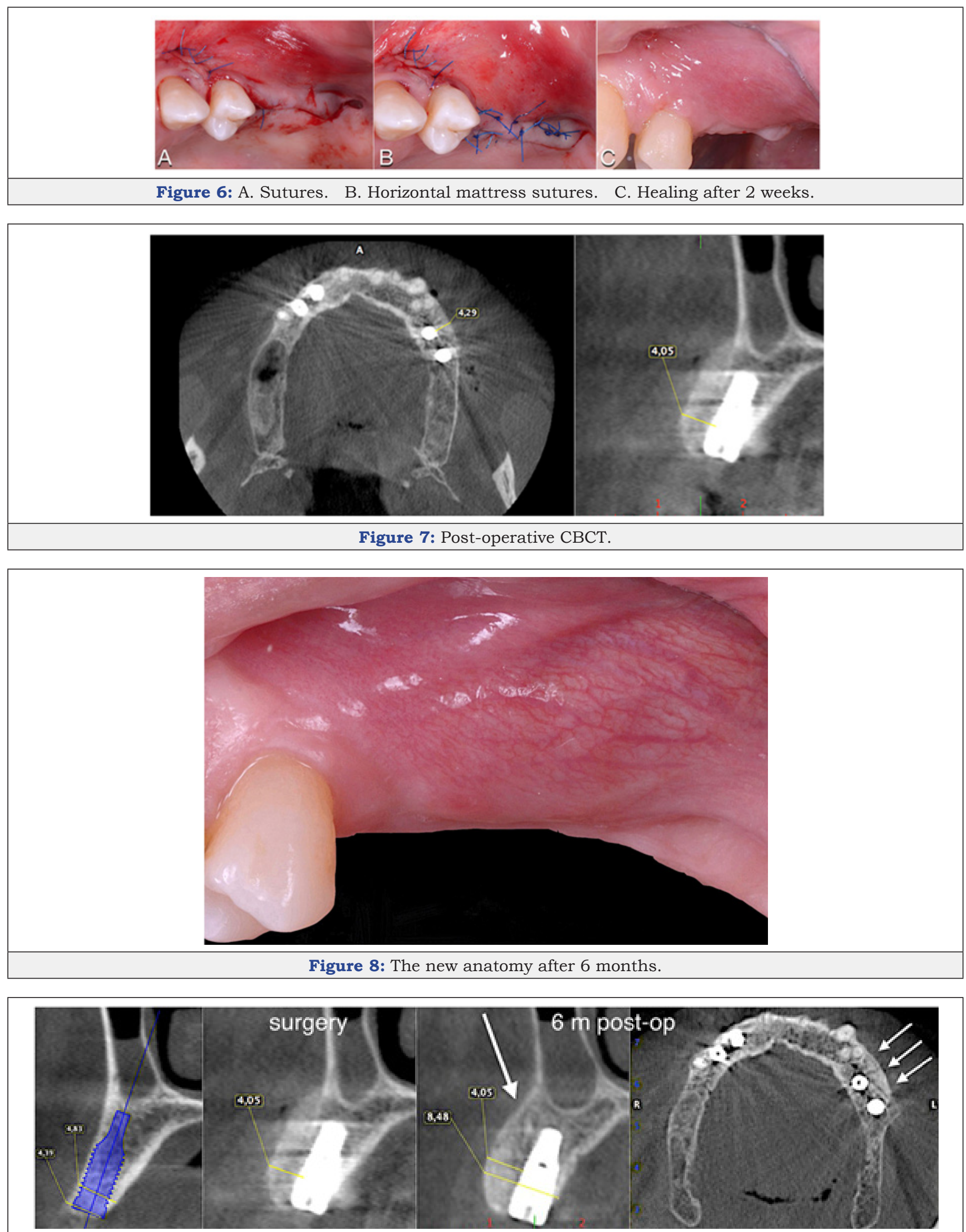

Figure 9: Horizontal increment of 4.05mm. Initial mineralization of newly formed bone (arrows). 

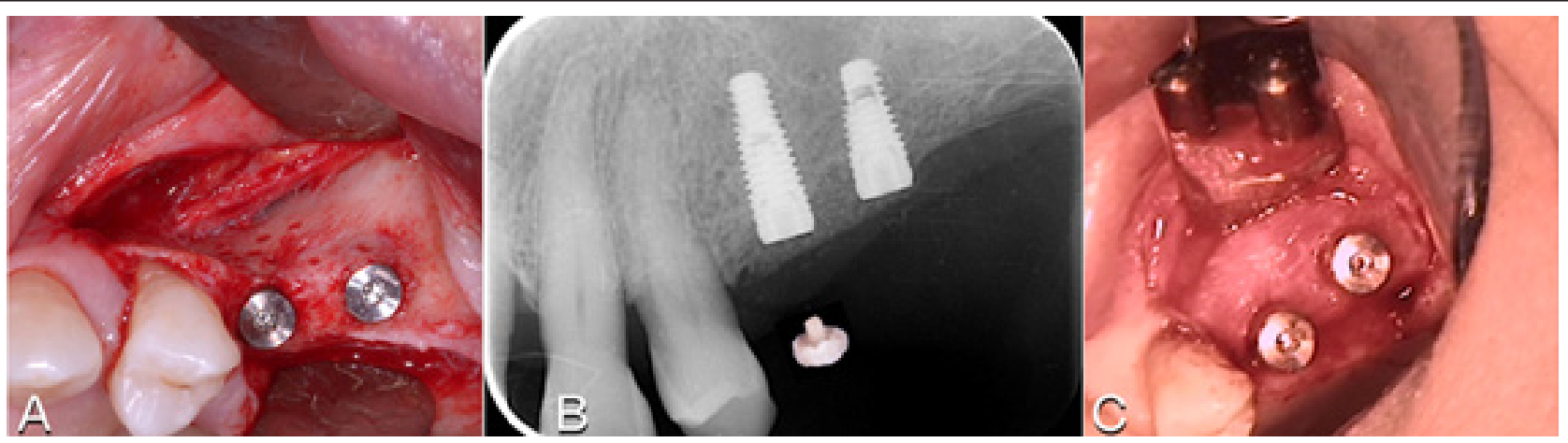

Figure 10: A. Concavity at baseline. B. Hard tissue removed from above the cover screw. C. Volume and convexity at re-entry.

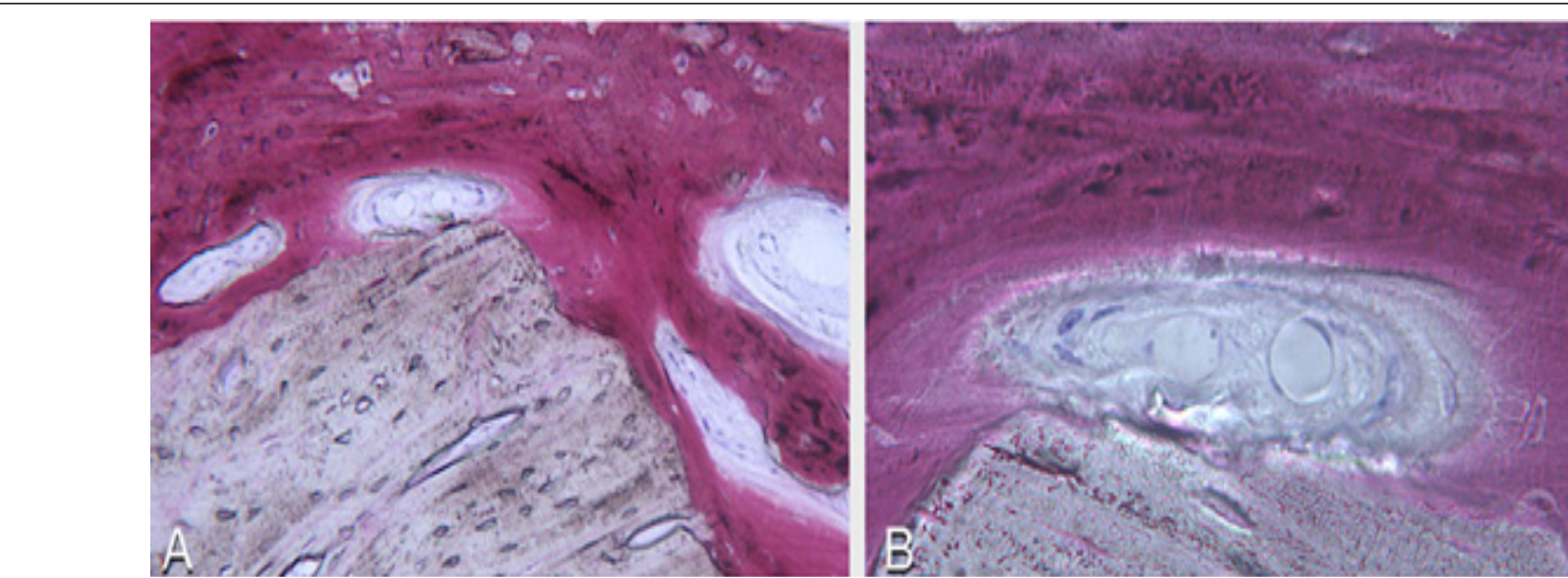

Figure 11: Courtesy of Prof. Giovanna Iezzi (University of Chieti-Pescara)

A. 100x: Particle of residual biomaterial in close contact with new mineralized trabecular bone and some osteoid matrix. No necrotic and inflammatory cells.

B. 200x: Particle of residual biomaterial in close contact with new mineralized trabecular bone and osteoid matrix in peripheral mineralization. Inside active osteoblasts and new blood vessels are visible.

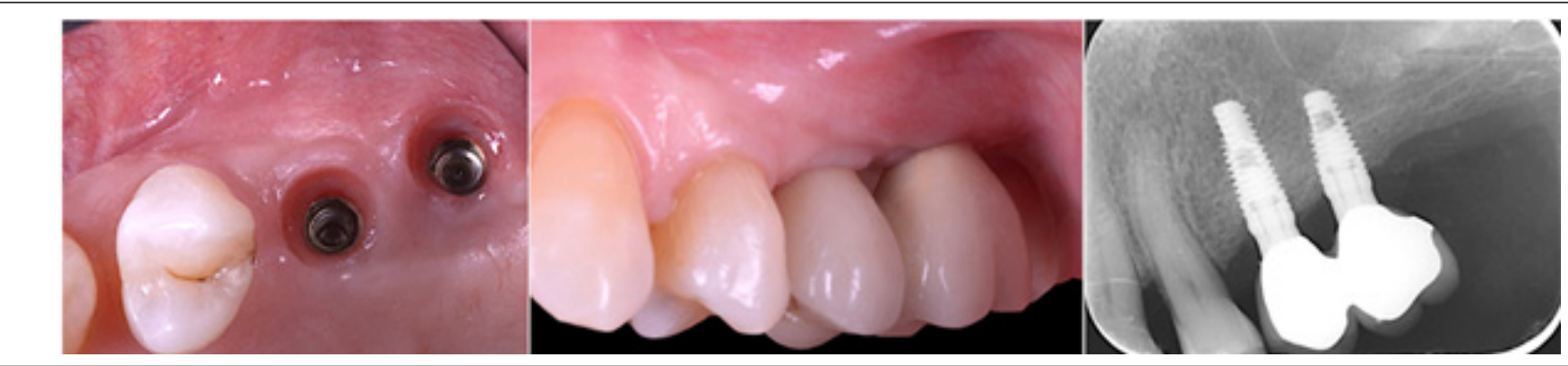

Figure 12: Prosthetic restoration.

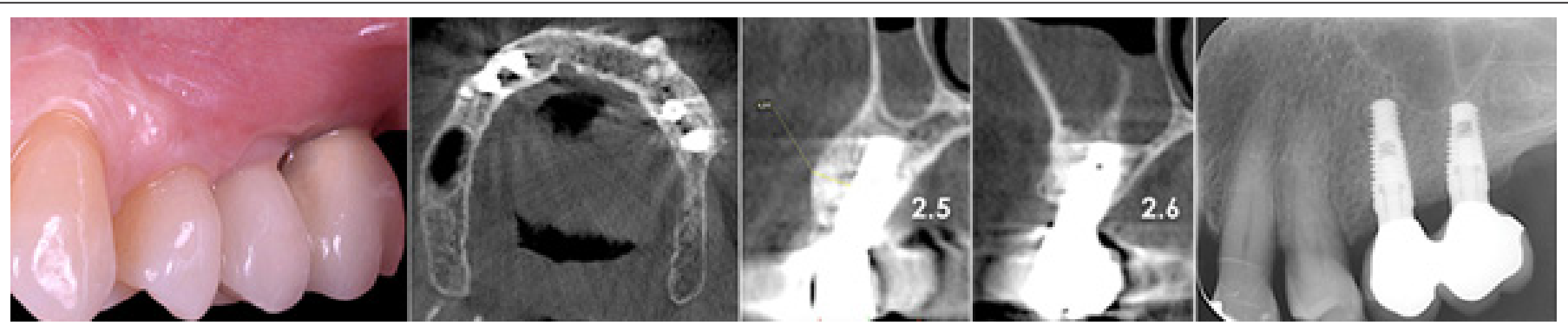

Figure 13: Maintenance of horizontal gain $(4.05 \mathrm{~mm})$. No bone loss at implant platforms. 

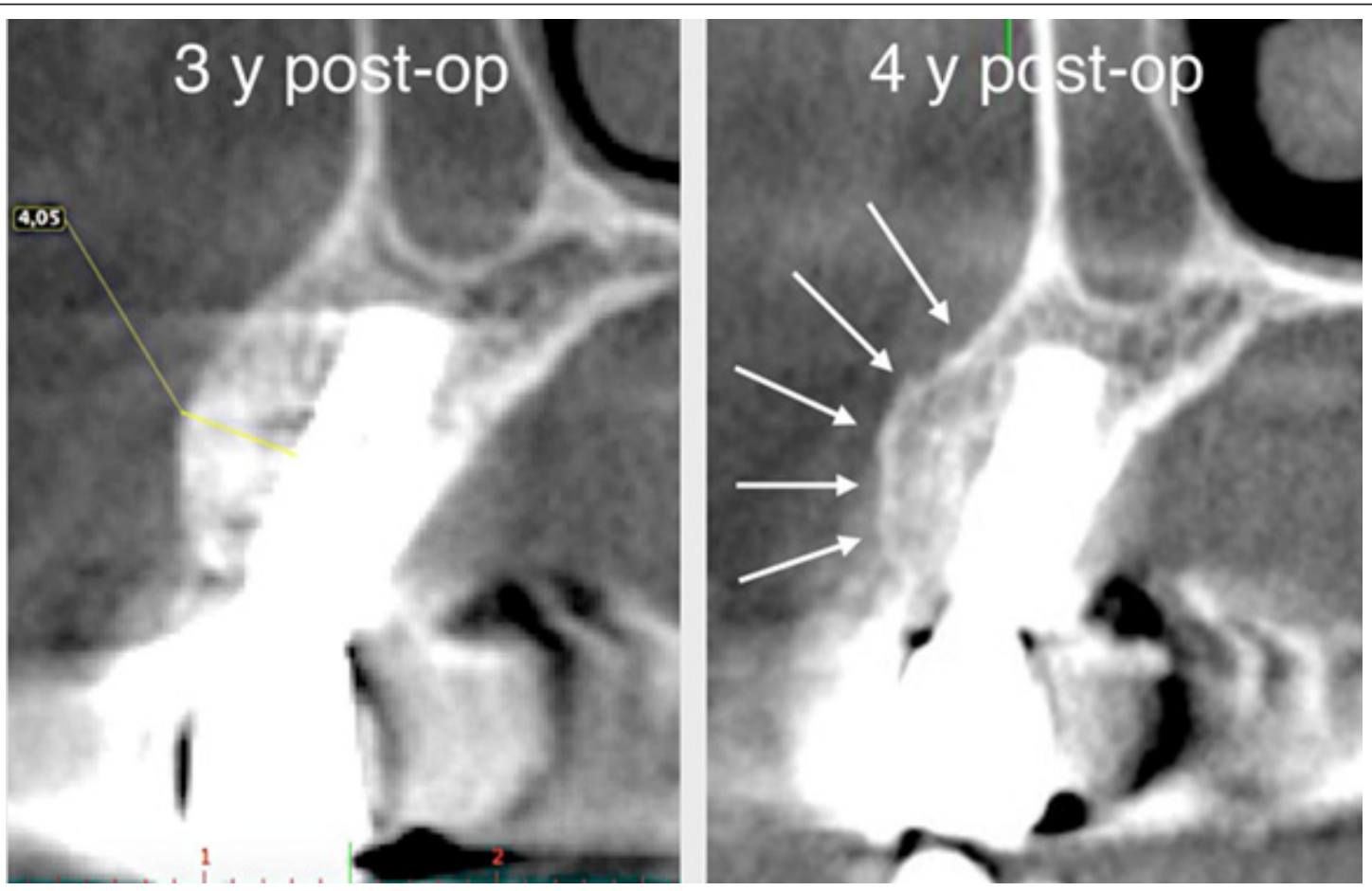

Figure 14: Four year post-op the graft is almost completely resorbed. The mineralization of newly formed bone is evident (arrows).
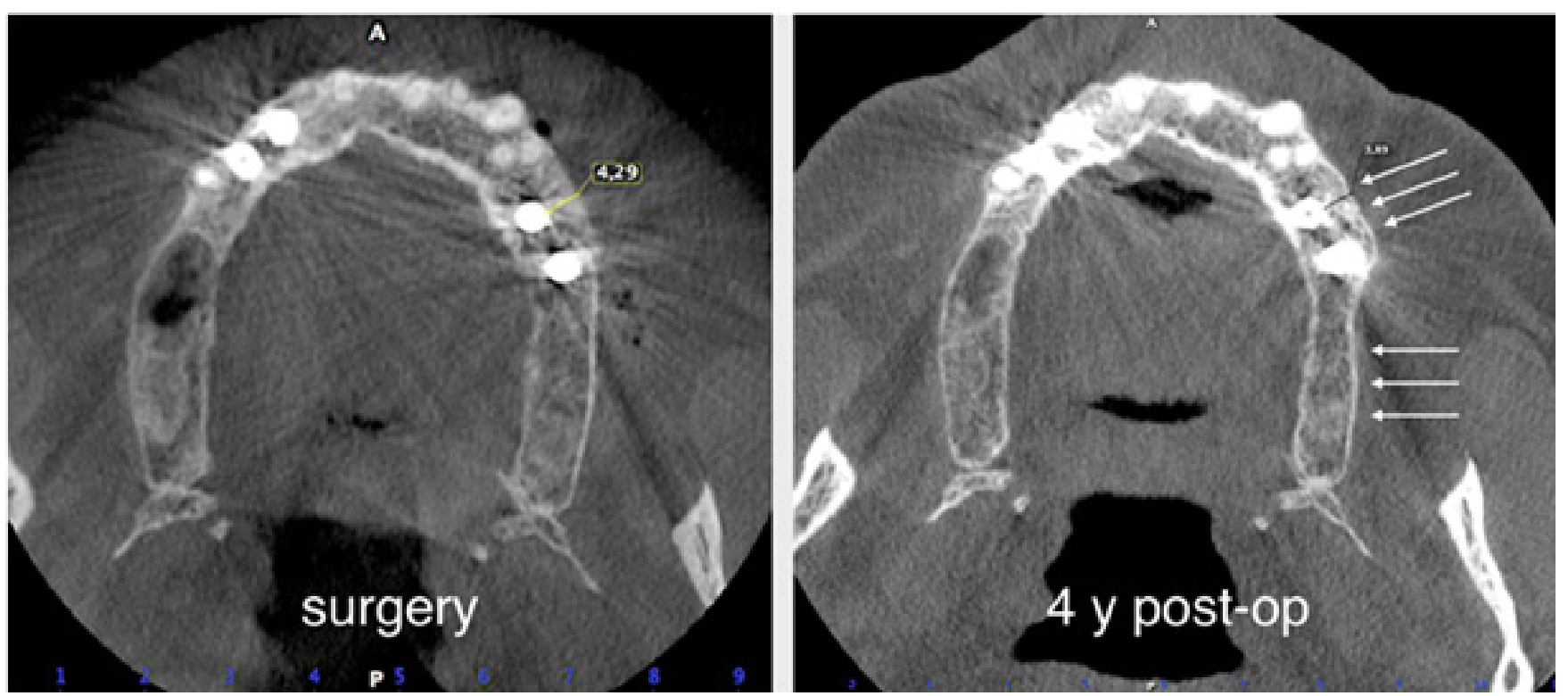

Figure 15: The new cortical bone is comparable to that of native bone (arrows).

\section{Case Presentation II}

A male patient 40 years old, affected by periodontitis grade IV referred to the clinic looking for a solution for his edentulous ridges. The CBCT showed a severely resorbed ridge at some points only one mm thick. Patient was in good health and accepted to undertake GBR in all edentulous areas by mean of the F.I.R.S.T. technique. The indication in this specific case was justified by the severe horizontal resorption, using some kind of 'sticky bone' would enhance the chances to obtain stability of the graft and good support for the cortical lamina placed on top of the augmented area. After local anesthesia with Articaine 1.200.000 full thickness buccal and lingual flaps were elevated to display what was already known from the CBCT (Figure 16). With a piezo surgical device, the buccal cortical bone was perforated in several different points to

1. Favor some bleeding from the marrow spaces.

2. Create some niches in the ridge in order to achieve stability of the bone graft (Figure 17). 

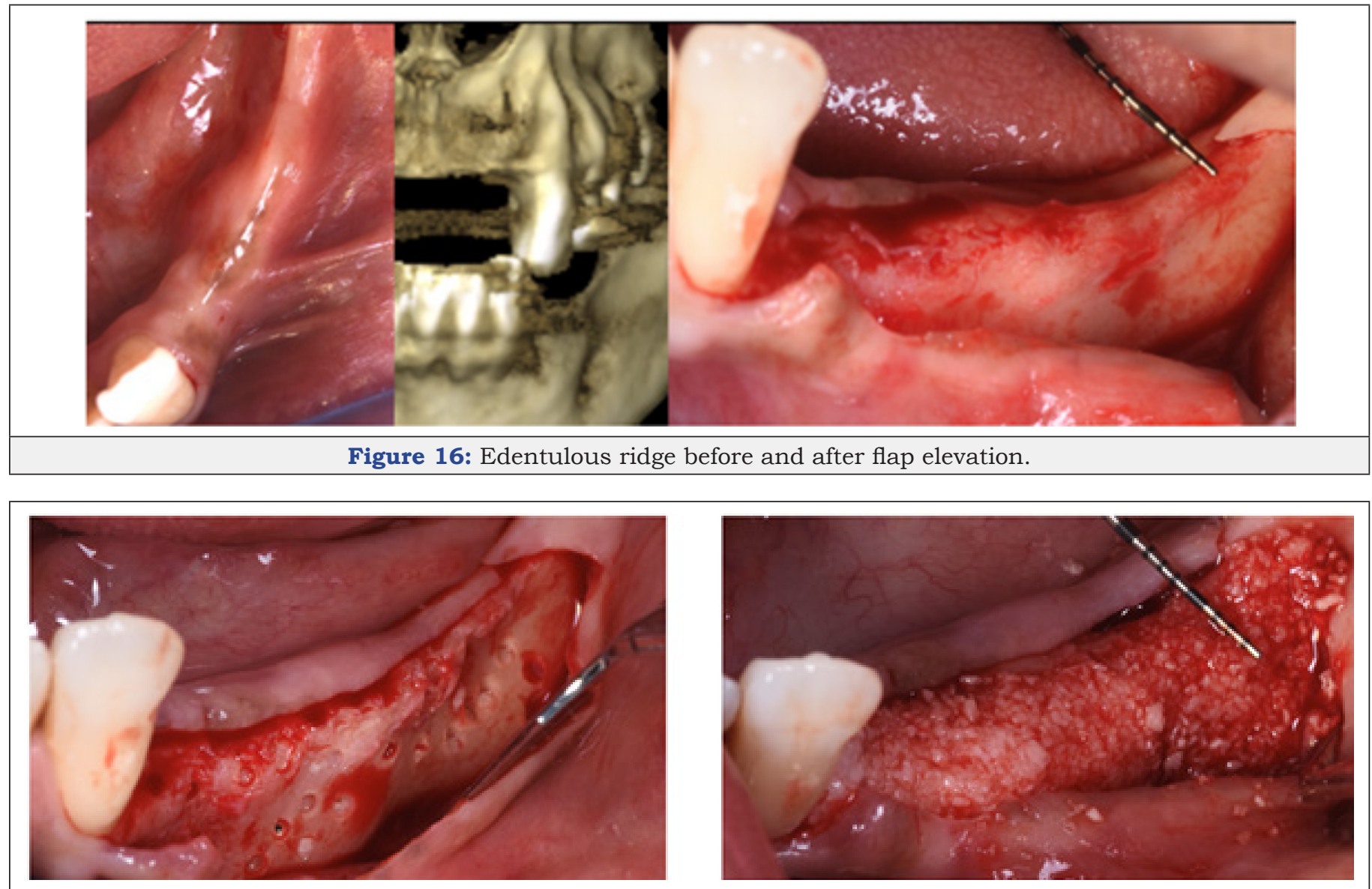

Figure 17: The intra-cortical holes guaranty further stability to the graft.

The protocol used to hydrate the cortico cancellous porcine xenograft (GenOs Osteobiol, Tecnoss, Coazze, Italy) was the same previously described, the use of the FS eases the stability and stickiness of the graft that adheres well to the edentulous ridge now measuring $5 \mathrm{~mm}$ in width. The addition of the cortical lamina glued by means of FS to a well set bone graft further increases the size of the ridge (Figure 18). In this specific case was important to overcontour the regeneration anticipating some kind of remodeling due to the fact that the patient was using a removable partial denture.
Because of his edentulism it was not possible to restrain the patient from the use of his denture, for this reason the augmentation provided an overcorrection of the defect anticipating some kind of remodeling with the use of the denture (Figure 19). Six months later it was possible to insert $3.4 \mathrm{~mm}$ diameter implants in the ridge that at baseline was $1 \mathrm{~mm}$ thick, the new ridge was now $5 \mathrm{~mm}$ wide. The two implants were restored with a long term temporary restoration (acrylic re-inforced with Kevlar) and three years after loading everything remained stable (Figure 20-23).

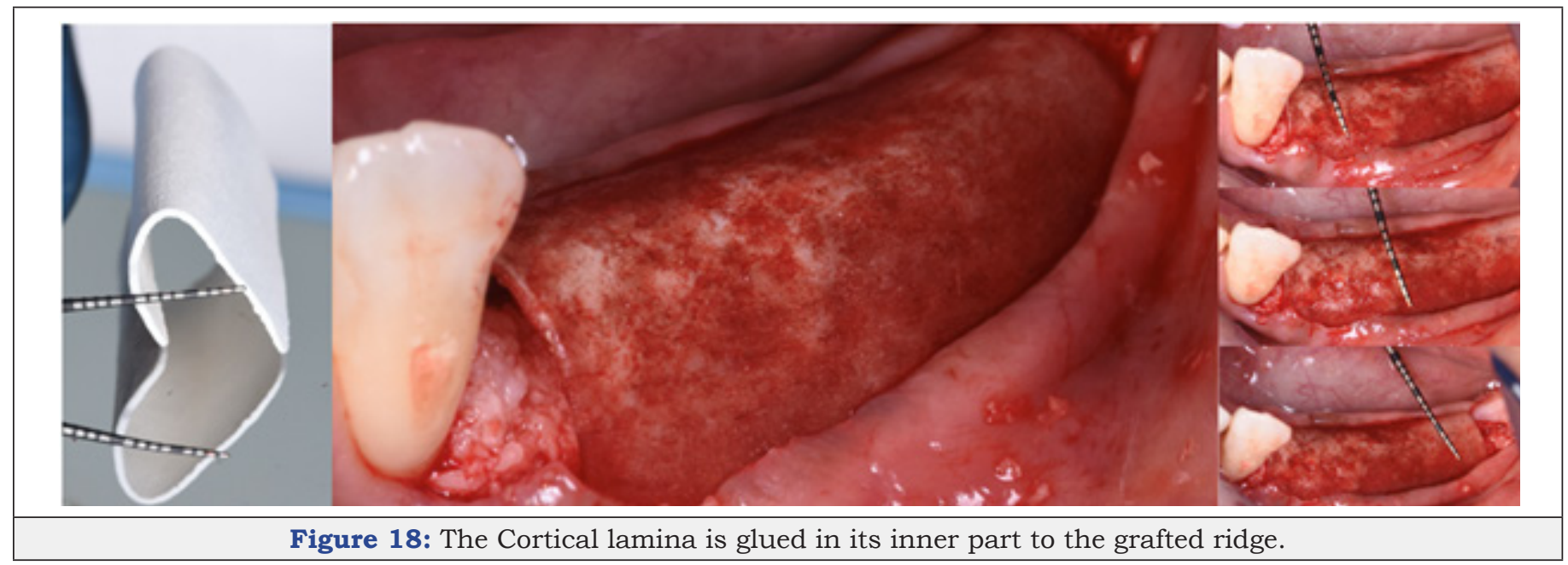



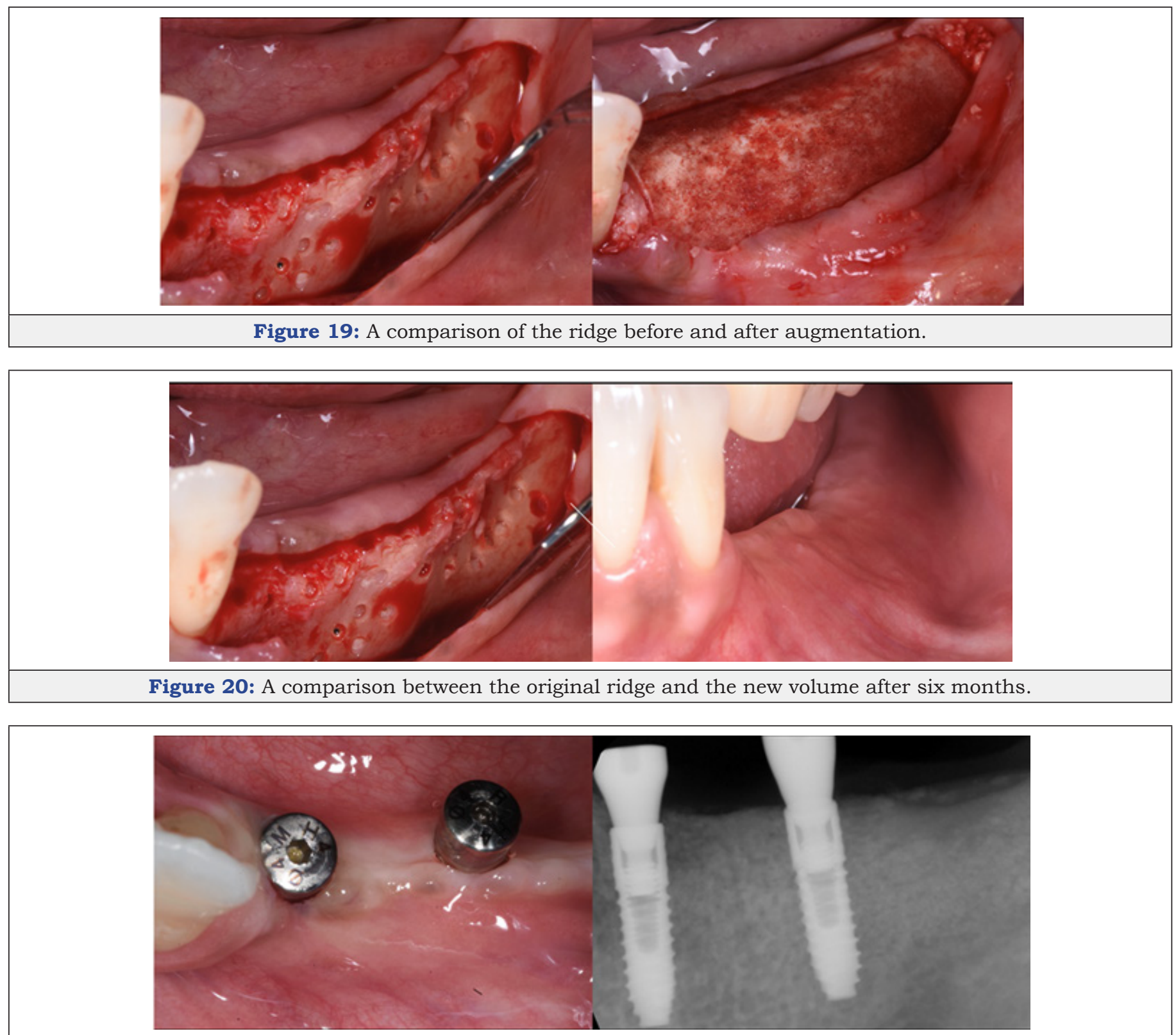

Figure 21: Standard diameter $3.4 \mathrm{~mm}$ implants placed six months after augmentation.

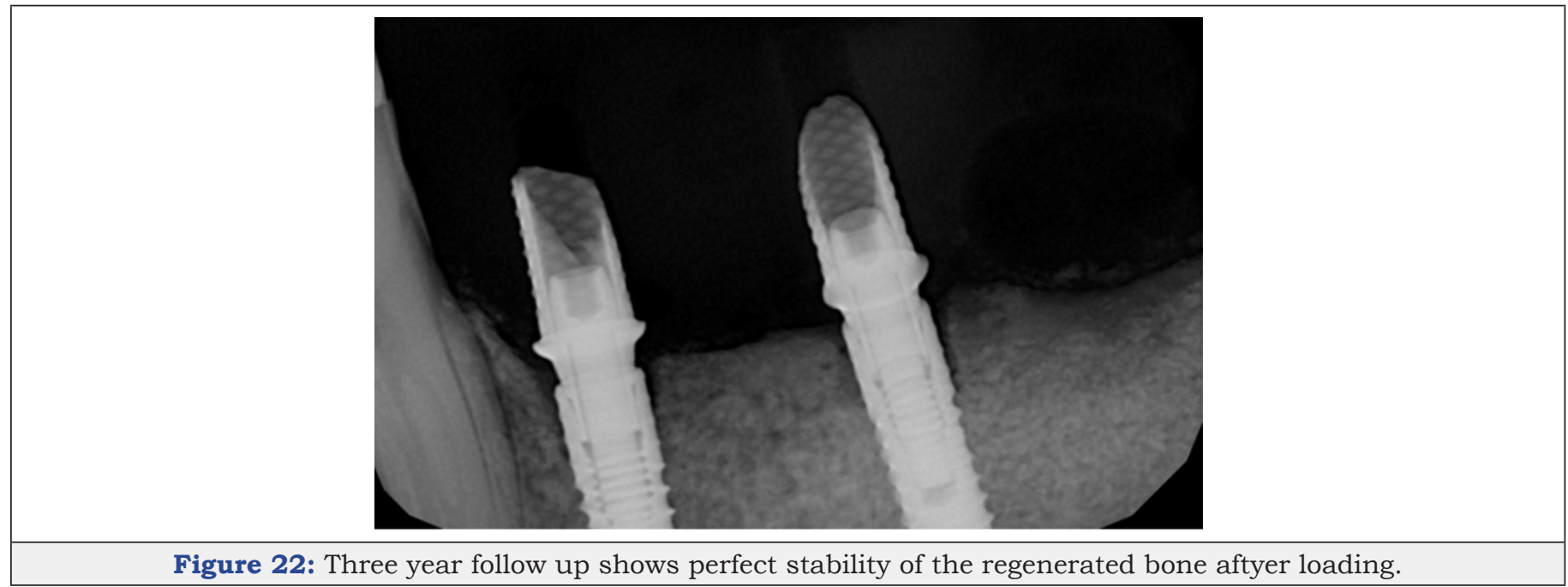

Figure 22: Three year follow up shows perfect stability of the regenerated bone aftyer loading. 


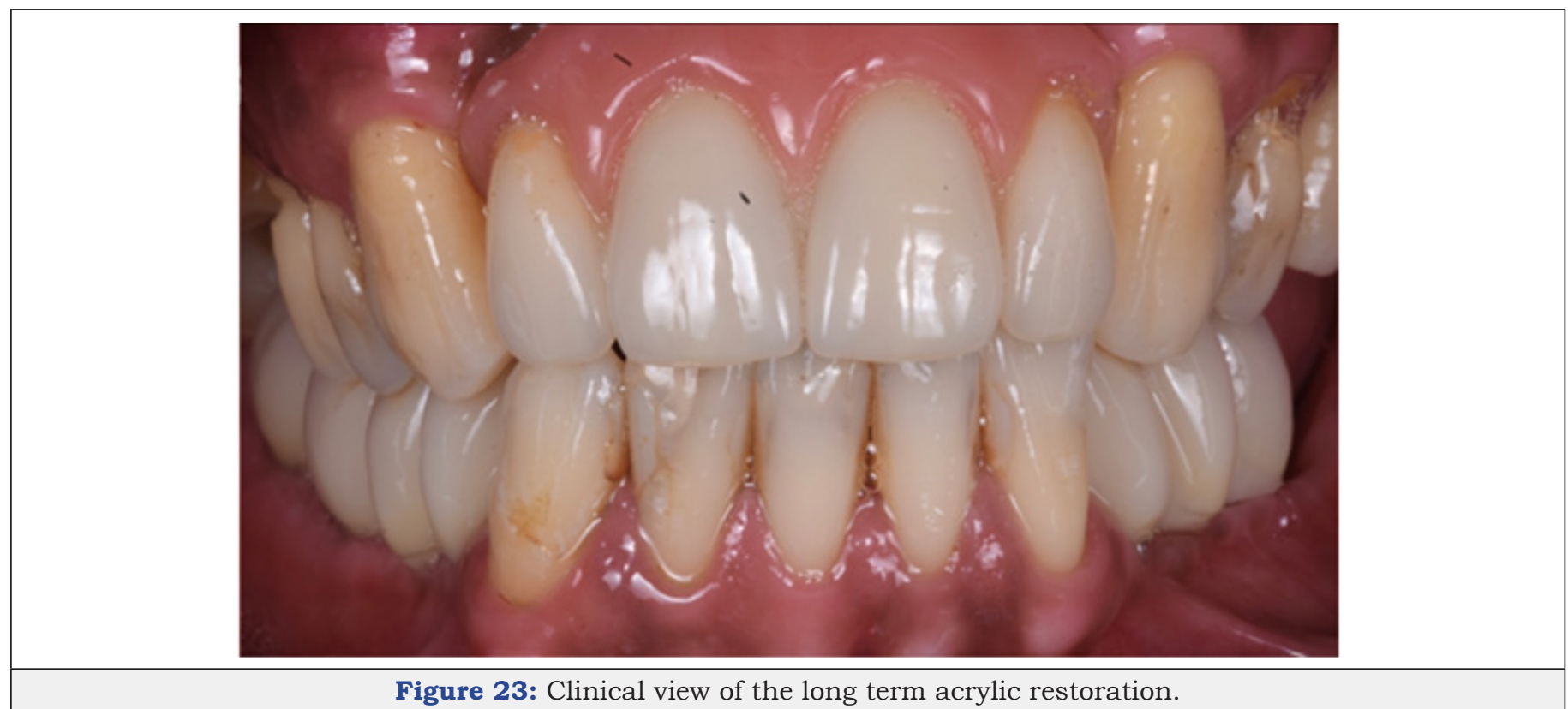

\section{Conclusion}

Guided bone regeneration is a well understood procedure to use in cases of severe bone deformities. The key factors for success of this procedure are : biocompatibility, tissue integration, space provision, cell occlusivity and clinical handling. Stability of the bone graft as well as stability of the protecting barrier membrane represents probably one of the cornerstones of this technique. Creating a very stable system represents a very good pre-requisite for success. The addition of fibrin sealant to the bone graft and the addition of the sealant to a bone membrane (gluing it to the local anatomy), proved in the two cases to be successful in

\section{Favoring bone regeneration and}

2. Providing a stable environment for osseointegration.

Further studies will be necessary to confirm the success of this modification that from these two case report appears very promising.

\section{References}

1. Wepner F, Fries R, Platz H (1982) The use of the fibrin adhesion system for local hemostasis in oral surgery. J Oral Maxillofac Surg 40(9): 555558.

2. Pini Prato GP, Cortellini P, Clauser C (1988) Fibrin and fibronectin sealing system in a guided tissue regeneration procedure. A case report. J Periodontol 59(10): 679-683.

3. Wittkampf AR (1989) Fibrin glue as cement for HA-granules. J Craniomaxillofac Surg 17(4): 179-181.

4. Hotz G (1991) Alveolar ridge augmentation with hydroxylapatite usung fibirn sealant for fixation. Part I: An experimental study. Int J Oral Maxillofac Surg 20(4): 204-207.

5. Hotz G (1991) Alveolar ridge augmentation with hydroxylapatite usung fibirn sealant for fixation. Part II: Clinical application. Int J Oral Maxillofac Surg 20(4): 208-213.

6. Corrente G, Abundo R, Cardaropoli G, Martuscelli G, Trisi P (1997) Supracrestal bone regeneration around dental implants using a calcium carbonate and a fibrin-fibronectin sealing system: clinical and histologic evidence. Int J Periodontics Restorative Dent 17(2): 170-181.

7. Cardaropoli D, Gaveglio L, Cardaropoli G (2013) Vertical ridge augmentation with a collagen membrane, bovine bone mineral and fibrin sealer: clinical and histologic findings. Int J Periodontics Restorative Dent 33(5): 583-589.

8. Le Guéhennec L, Layrolle P, Daculsi G (2004) A review of bioceramics and fibrin sealant. Eur Cell Mater 8: 1-10.

9. Schwarz N, Redl H, Zeng L, Schlag G, Dinges HP, et al. (1993) Early osteoinduction in rats is not altered by fibrin sealant. Clin Orthop Relat Res (293): 353-359.

10. Abiraman S, Varma HK, Umashankar PR, John A (2002) Fibrin glue as an osteoinductive protein in a mouse model. Biomaterials 23(14): 30233031.

11. Le Nihouannen D, Saffarzadeh A, Aguado E, Goyenvalle E, Gauthier O, et al. (2007) Osteogenic properties of calcium phosphate ceramics and fibrin glue based composites. J Mater Sci Mater Med 18(2): 225-235.

12. Sahni A, Francis CW (2000) Vascular endothelial growth factor binds to fibrinogen and fibrin and stimulates endothelial cell proliferation. Blood 96(12): 3772-3778.

13. Rajangam T, An SS (2013) Improved fibronectin-immobilized fibrinogen microthreads for the attachment and proliferation of fibroblasts. Int J Nanomedicine 8: 1037-1049.

14. Linsley C, Wu B, Tawill B (2013) The effect of fibrinogen, collagen type $\mathrm{i}$, and fibronectin on mesenchymal stem cell growth and differentiation into osteoblasts. Tissue Eng Part A 19(11-12): 1416-1423.

15. Rybarczyk BJ, Lawrence SO, Simpson-Haidaris PJ (2003) Matrixfibrinogen enhances wound closure by increasing both cell proliferation and migration. Blood 102(12): 4035-4043.

16. Rossi R, Foce E (2019) Reconstruction of a horizontal and vertical defect using the cortical lamina technique. Med Research Arch 7(11).

17. Rossi R, Rancitelli D, Poli PP, Rasia da Polo M, Nannmark U, et al. (2016) The use of collagenated porcine cortical lamina in the reconstruction of alveolar ridge defects: a clinical and histological study. Minerva Stomatol 65(5): 257-268.

18. Wang HL, Boyapati L (2006) "PASS" principles for predictable bone regeneration. Implant Dent 15(1): 8-17. 
19. El Nahass H, Naiem SN (2015) Analysis of the dimensions of the labial bone wall in the anterior maxilla: a cone-beam computed tomography study. Clin Oral Implants Res 26(4): e57-e61.

20. Carmagnola D, Berglundh T, Lindhe J (2002) The effect of a fibrin glue on the integration of Bio-Oss with bone tissue. An experimental study in labrador dogs. J Clin Periodontol 29(5): 377-383.
21. Traini T, Piattelli A, Caputi S, Degidi M, Mangano C, et al. (2015) Regeneration of human bone using different bone substitute biomaterials. Clin Implant Dent Relat Res 17(1): 150-162. 